\title{
High-energy mid-infrared sub-cycle pulse synthesis
}

\author{
Kyung-Han Hong ${ }^{*}$, Peter Krogen, Tobias Kroh, and \\ Franz X. Kärtner \\ Research Laboratory of Electronics, \\ Massachusetts Institute of Technology \\ Cambridge, Massachusetts, USA \\ kyunghan@mit.edu
}

\author{
Houkun Liang \\ Singapore Institute of Manufacturing Technology \\ 2 Fusionopolis Way, Singapore
}

\begin{abstract}
We present the carrier-envelope phase-stable mid-infrared sub-cycle pulses, synthesized from an optical parametric amplifier covering the $2.5-9.0 \mu \mathrm{m}$ range. The strong-field applications in solids and nano-structures are discussed.
\end{abstract}

mid-infrared lasers; optical parametric amplifiers; optical waveform synthesis; high-harmonic generation

\section{INTRODUCTION}

High-energy, sub-cycle, mid-infrared (mid-IR) pulses with long-term carrier-envelope phase (CEP) stability can provide unique opportunities of exploring phase-sensitive strong-field light-matter interactions in atoms, molecules, and solids. In the mid-IR wavelength, the ponderomotive energy of laser pulses is dramatically increased (versus the visible/near-infrared) and therefore, the Keldysh parameter is much smaller than unity even at relatively modest laser intensities. This enables to study the strong-field sub-cycle electron dynamics in solids without damage via high-harmonic generation (HHG) $[1,2]$. One can also control the electron emissions from nano-devices in the sub-cycle time scale ${ }^{5-7}$. These efforts are opening a great opportunity towards "petahertz electronics".

Pulse synthesis is a very powerful method to overcome the bandwidth limit of conventional laser amplifiers. One can generate and manipulate an optical bandwidth more than one octave by coherently combining ultrashort pulses covering different spectral windows with controlled phase. Single- or sub-cycle pulse synthesizers in the visible-to-near-infrared (IR) spectral region have been demonstrated by coherent multiplexing of pulses from fiber lasers, supercontinuum [3], and optical parametric chirped-pulse amplifiers (OPCPA) [4]. In the mid-IR range, four-wave mixing through filamentation in gas has been adapted to demonstrate the conical emission of phase-stable sub-cycle pulses [5] and a technique that cascades difference-frequency generation (DFG), spectral broadening, and chirp-compensation [6] have been demonstrated. However, the energy scalability is limited to only $\sim 1 \mu \mathrm{J}$ level in those techniques.

In this work, we present a multi-GW, sub-cycle in-line pulse synthesizer based on a mid-IR optical parametric amplifier (OPA), pumped by CEP-stable, $2.1 \mu \mathrm{m}$ femtosecond pulses. The signal and idler combined spectrum spans from 2.5 to $9.0 \mu \mathrm{m}$, which covers the whole midwave-infrared (MWIR) region. We coherently synthesize the passively CEP-stable few-cycle signal and idler pulses to generate $33 \mu \mathrm{J}, 0.88$-cycle (12.4 fs), pulses centered at $\sim 4.2 \mu \mathrm{m}$, which is further energy scalable. The in-line synthesis of the CEP-stable sub-cycle pulse is realized through a type-I collinear OPA with minimal temporal walk-off in a thin $\mathrm{CdSiP}_{2}$ (CSP) crystal, which also supports a phase-matching bandwidth greater than one octave at the idler wavelength. The temporal profile of synthesized pulse is characterized using a cross-correlation frequencyresolved optical gating (XFROG) apparatus. The good CEP stability of signal and idler pulses is individually confirmed. We also successfully showed HHG in thin solid materials, such as silicon, $\mathrm{ZnO}$, and $\mathrm{NiO}$, using this mid-IR source. Our demonstration offers an energy scalable and technically simple platform of laser sources generating CEP-stable sub-cycle pulses in the whole MWIR region for investigating phasesensitive strong-field interactions.

\section{MID-IR SUB-CYCLE PULSE GENERATION}

\section{A. Experimental Setup}

The mid-IR sub-cycle source is based on a 2.1- $\mu$ m-pumped OPA, as shown in Fig. 1. An octave-spanning Ti:sapphire oscillator provides the seed for the 2.1- $\mu$ m OPCPA through an intrapulse DFG stage which ensures passive CEP stabilization. The kHz, multi-mJ, CEP-stable, 2.1- $\mu \mathrm{m}$ OPCPA [7] serves as the pump of the mid-IR OPA. A $20 \mu \mathrm{J}$ portion of the $2.1-\mu \mathrm{m}$ pump is split for white light generation (WLG) in a 6-mmthick $\mathrm{BaF}_{2}$ plate [8] as the signal of the mid-IR OPA. A 1.1mm-thick CSP is chosen for the type-I parametric conversion for its large nonlinear coefficient, broad phase-matching bandwidth, and high damage threshold pumped by $2.1-\mu \mathrm{m}$ pulses. The pump beam size is $6 \mathrm{~mm}$ in diameter and intensity is $150 \mathrm{GW} / \mathrm{cm}^{2}$ at $800 \mu \mathrm{J}$ of pump energy. The output pulse from the mid-IR OPA together with a $\sim 10 \mu \mathrm{J}, 2.1-\mu \mathrm{m}$ pulse split from the pump which serves as the reference beam are sent into the XFROG for the temporal characterization.

While the CEP-stable pump enables to provide the CEPstable signal pulse via WLG, the CEP of the idler pulse is also passively stabilized by DFG-like parametric process between the WLG signal and the pump pulses, regardless of the CEP stability of the pump. Therefore, the long-term CEP stability of both signal and idler pulses is ensured without active stabilization pulse compression techniques. 


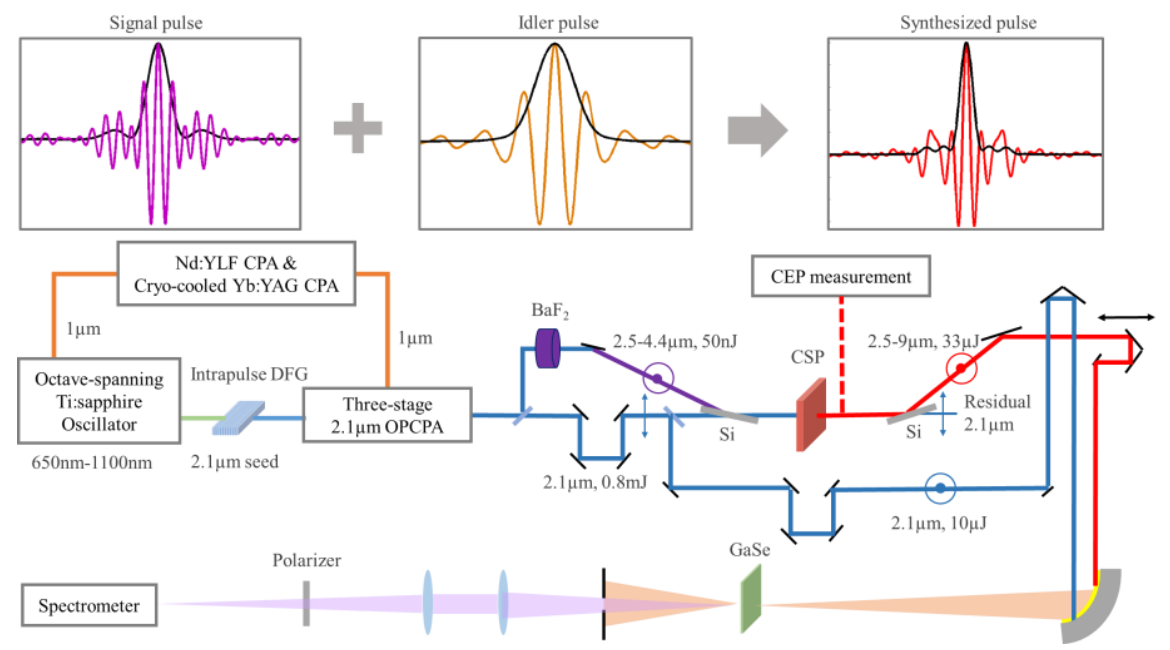

Fig. 1 The schematic of the high-energy phase-stable sub-cycle mid-IR OPA. CPA: chirped-pulse amplifier. Polarizations of the beams are marked by doubleheaded arrows and concentric circles. 300- $\mu \mathrm{m}$ thick Si wafers at Brewster angle are used as polarization beam splitter and beam combiner to transmit the $2.1-\mu \mathrm{m}$ pump pulse and reflect the signal and idler pulses. The synthesized pulses and a branch of $2.1-\mu \mathrm{m}$ reference pulses are sent into XFROG with $30-\mu \mathrm{m}$ thick GaSe nonlinear crystal. The synthesis of a sub-cycle mid-IR pulse from coherently combining the sub-2-cycle signal and idler pulses is shown conceptually on the top of the figure.

\section{B. Characterization of OPA pulses}

Fig. 2(a) shows the mid-IR spectrum of the signal pulse which is a part of the supercontinuum from the WLG. We obtain $\sim 50 \mathrm{~nJ}$ of pulse energy within the spectral window of 2.5- $4.4 \mu \mathrm{m}$. The spectra of the amplified signal and idler with $0.8 \mathrm{~mJ}$ pump energy are shown in Fig. 2(b), spanning from 2.5 to $9.0 \mu \mathrm{m}$. It is worth noting that the long-wavelength component of the pump at $2.2-2.3 \mu \mathrm{m}$ gives excellent phase matching in the spectral range of 4-8 $\mu \mathrm{m}$. Therefore, the 2.2 $2.3 \mu \mathrm{m}$ frequency component of our broadband pump is very helpful to achieve the octave-spanning parametric conversion. $33 \mu \mathrm{J}$ output energy from the mid-IR OPA at $0.8 \mathrm{~mJ}$ pump is demonstrated as shown in Fig. 2(c), of which there is a $12 \mu \mathrm{J}$ idler pulse spanning from 4.4 to $9.0 \mu \mathrm{m}$. The conversion efficiency to the synthesized pulse is $\sim 6 \%$ considering the Fresnel reflection of the pump at the uncoated CSP crystal. While the available pump energy is higher than $2 \mathrm{~mJ}$, the OPA stage has been designed at $\sim 1 \mathrm{~mJ}$ of pump energy in this work because of the energy loss from metallic mirrors (13 bounces). The near-Gaussian signal and idler beam profiles, measured with a pyroelectric camera (PyroCam III, Spiricon) are presented in Fig. 2(d) and (e), respectively. The idler spectrum can be even broader (up to $10 \mu \mathrm{m}$ along with a stronger signal pulse), if we use a higher $2.1 \mu \mathrm{m}$ pulse energy for WLG. However, we limit the energy to $\sim 50 \mathrm{~nJ}$ within the signal bandwidth because the CEP of the signal generated by WLG is found to be less stable at higher energy.

The temporal profile of the $2.1-\mu \mathrm{m}$ pulse, which serves both as the pump of the mid-IR OPA and as reference of the XFROG, is characterized using a second-harmonic generation (SHG) FROG device. The pump pulse whose spectrum spans from $1.8 \mu \mathrm{m}$ to $2.3 \mu \mathrm{m}$ is within $5 \%$ of its transform limit, with a pulse width of $26 \mathrm{fs}$ in FWHM. The signal pulse from WLG has a slight self-compression in $\mathrm{BaF}_{2}$ due to small negative dispersion at $2.1 \mu \mathrm{m}$. The pulse duration of the amplified signal is measured as $\sim 20$ fs using the SHG FROG.

We also investigate the temporal profile of the idler pulse which already contains an octave-spanning spectral content. The amplified idler pulse is independently characterized using a home-built mid-IR second-order interferometric autocorrelator (IAC). An uncoated 1-mm-thick ZnSe plate is employed as a beam splitter, and a $4.5-\mu \mathrm{m}$ long-pass filter is used to isolate the idler pulse from the signal and any residual pump. The anomalous dispersion from the $\mathrm{ZnSe}$ plate is well compensated by the normal dispersion from the Ge substrate of the long-pass filter, however there is approximately $8000 \mathrm{fs}^{3}$ of uncompensated third-order dispersion (TOD) in this configuration. The measured IAC that the idler is nearly transform limited with a pulse width less than 1.5 optical cycles, centered at $6.4 \mu \mathrm{m}$. $\sim 31 \mathrm{fs}$ pulse width is deconvoluted assuming the idler pulse is in the Gaussian profile. The CEP
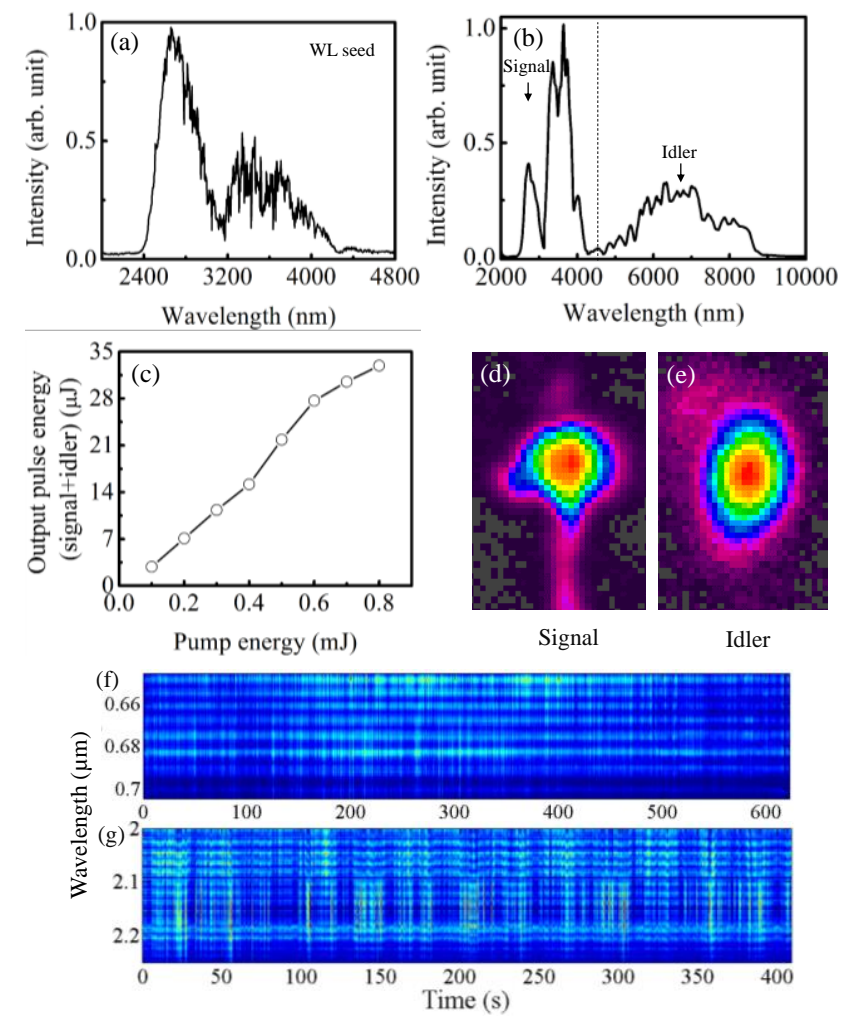

Fig. 2 (a) The signal spectrum from WLG at $\mathrm{BaF}_{2}$ with a $2400 \mathrm{~nm}$ longpass filter. (b) The measured output spectrum of the mid-IR OPA. The dotted line separates the signal and idler spectra. (c) The output pulse energy of the mid-IR OPA vs. pump energy. The far-field beam profile of the amplified signal (d) and idler (e) beams. (f) self-referencing f-3f SI of signal pulses, and ( $\mathrm{g}$ ) cross-referencing f-3f SI of idler pulses.

Supported by U.S. AFOSR (FA9550-12-1-0499, and FA9550-14-1-0255) and CFEL-DESY, Germany 
stability of the signal and idler pulses is measured using the self-referencing and cross-referencing $f$ - $3 f$ spectral interferometry (SI), as shown in Figs. 2(f) and (e), respectively. The shot-to-shot fluctuations of $\sim 250 \mathrm{mrad}$ are measured for both pulses over 6-10 minutes. Therefore, the sub-two-cycle signal and idler pulses are already usable for phase-sensitive strong-field experiments as a stand-alone source and can be coherently synthesized if they are temporally overlapped.

\section{Characterization of synthesized sub-cycle pulses}

The temporal profile of the synthesized pulse is characterized with XFROG. The time delay between the signal and pump pulses is optimized, such that the shortest duration is obtained while the amplified energy is maintained at maximum. The measured and retrieved XFROG traces are shown in Fig. 3(a) and (b), respectively, with 1.8\% FROG error. The retrieved spectrum shown in Fig. 3(c) agrees well with the measured spectrum in Fig. 2(b). The synthesized pulse has a near-transform-limited main peak and rippling wings as shown in Fig. 3(d), attributed to the interference of the signal and idler pulses. The synthesized pulse duration is measured as $\sim 12.4 \mathrm{fs}$ in FWHM centered at $\sim 4.2 \mu \mathrm{m}$, corresponding to 0.88 optical cycle. With the energy portion into the main pulse of $\sim 70 \%$ the peak power reaches $\sim 1.9 \mathrm{GW}$. We obtain similarly broad spectra and high energy from an OPA with a $0.5-\mathrm{mm}-$ thick ZGP crystal. However, due to a relatively large temporal walk-off of $\sim 11 \mathrm{fs}$ between the signal and idler, this crystal is less favorable than the CSP crystal for the sub-cycle pulse synthesis.
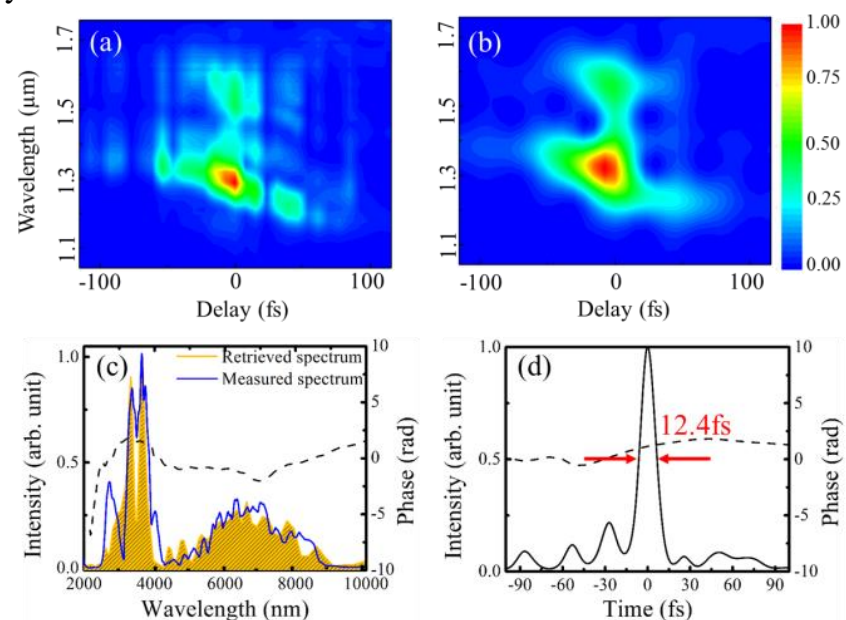

Fig. 3 (a) The temporal profile characterization of the synthesized mid-IR pulse using XFROG. The measured (a) and the retrieved (b) XFROG traces. The FROG error is $1.8 \%$. The retrieved spectral (c) and temporal (d) intensity profiles of the synthesized pulse. The dotted curves are the retrieved phase.

The scaling of energy and peak power is relatively straightforward by adding more OPA stages with higher pump energy. The availability of Joule-level ps Yb-doped lasers ensures the energy scalability of our $2.1 \mu \mathrm{m}$ OPCPA to multiten $\mathrm{mJ}$ level, which can eventually increase the synthesized mid-IR pulse energy to multi-mJ. Advanced $\sim 2-3 \mu \mathrm{m}$ pump laser technologies [9] can be used to realize a relatively compact multi-mJ sub-cycle mid-IR laser source if the sub-50 fs duration can be accessed by these lasers via external pulse compression.

\section{APPLICATIONS TO STRONG-FIELD INTERACTIONS}

We have performed $\mathrm{HHG}$ in solid materials, such as silicon, $\mathrm{ZnO}$, and $\mathrm{NiO}$, using our sub-cycle mid-IR source. For example, with 200-nm-thick and 500-nm-thick silicon samples we have observed HHG up to $\sim 15^{\text {th }}$ order of $\sim 4.5 \mu \mathrm{m}$ center wavelength. Near-continuum harmonic spectra are observed due to the sub-cycle duration of the driver. By slightly broadening the pulse duration using a 0.5 -mm-thick silicon filter, we are able to observe distinct harmonic peaks including strong even harmonics induced by the non-sinusoidal waveform [10]. The strong-field electron emission from a nano-device [11] will also be discussed in the presentation.

\section{CONCLUSIONS}

We have demonstrated a high-energy, CEP-stable, subcycle, mid-IR pulse synthesizer based on an OPA covering the bandwidth from 2.5 to $9.0 \mu \mathrm{m}$. The synthesized pulse width is measured as $\sim 12.4 \mathrm{fs}$, corresponding to 0.88 optical cycle at $\sim 4.2 \mu \mathrm{m}$. The synthesized pulse energy of $33 \mu \mathrm{J}$ with a peak power of $\sim 1.9 \mathrm{GW}$ is obtained, which is already high enough for strong-field experiments, such as HHG in solids and electron tunneling emission from nano-structures.

\section{ACKNOWLEDGMENT}

We thank K. Zawilski and P. Schunemann at BAE Systems for providing the CSP and ZGP crystals and J. Moses for the initial contribution to this work. Z. Wang, H. Park, and L. DiMauro are highly appreciated for the collaboration about the HHG in solids. We thank Dr. Tino Lang for letting us use the $2+1$ dimensional nonlinear pulse propagation analyzer.

\section{REFERENCES}

[1] S. Ghimire et al., "Observation of high-order harmonic generation in a bulk crystal,” Nature Phys. 7 138-141 (2011).

[2] O. Schubert et al., "Sub-cycle control of terahertz high-harmonic generation by dynamical Bloch oscillations," Nature Photon. 8, 119 (2014).

[3] A. Wirth et al., "Synthesized light transients," Science 334, 195-200 (2011).

[4] S.-W. Huang et al., "High-energy pulse synthesis with sub-cycle waveform control for strong-field physics," Nature Photon. 5, 475-479 (2011).

[5] T. Fuji T., Y. Nomura, and H. Shirai, "Generation and Characterization of Phase-Stable Sub-Single-Cycle Pulses at $3000 \mathrm{~cm}^{-1}$," IEEE J. Sel. Top. Quantum Electron. 21, 8700612 (2015)

[6] E. A. Stepanov et al., "Solid-state source of subcycle pulses in the midinfrared," Phy. Rev. Lett. 117, 043901 (2016).

[7] K.-H. Hong et al., "Multi-mJ, kHz, $2.1 \mu \mathrm{m}$ optical parametric chirpedpulse amplifier and high-flux soft x-ray high harmonic generation," Opt. Lett. 39, 3145-3148 (2014).

[8] H. K. Liang et al., "Three-octave-spanning supercontinuum generation and sub-two cycle self-compression of mid-infrared filaments in dielectrics," Opt. Lett. 40, 1069-1072 (2015).

[9] S. Mirov et al., "Progress in Mid-IR Lasers Based on Cr and Fe Doped II-VI Chalcogenides (Invited paper)", IEEE Selected Topics in Quantum Electronics 21, 1601719 (2015)

[10] G. Vampa et al., "Linking high harmonics from gases and solids," Nature 522, 462 (2015)

[11] W. Putnam et al., "Optical-field-controlled photoemission from plasmonic nanoparticles," Nat. Physics (2016), doi:10.1038/nphys3978

Supported by U.S. AFOSR 
